\title{
Estimating Linguistic Complexity for Science Texts
}

\author{
Farah Nadeem and Mari Ostendorf \\ Dept. of Electrical Engineering \\ University of Washington \\ \{farahn, ostendor\}@uw.edu
}

\begin{abstract}
Evaluation of text difficulty is important both for downstream tasks like text simplification, and for supporting educators in classrooms. Existing work on automated text complexity analysis uses linear models with engineered knowledge-driven features as inputs. While this offers interpretability, these models have lower accuracy for shorter texts. Traditional readability metrics have the additional drawback of not generalizing to informational texts such as science. We propose a neural approach, training on science and other informational texts, to mitigate both problems. Our results show that neural methods outperform knowledge-based linear models for short texts, and have the capacity to generalize to genres not present in the training data.
\end{abstract}

\section{Introduction}

A typical classroom presents a diverse set of students in terms of their reading comprehension skills, particularly in the case of English language learners (ELLs). Supporting these students often requires educators to estimate accessibility of instructional texts. To address this need, several automated systems have been developed to estimate text difficulty, including readability metrics like Lexile (Stenner et al., 1988), the end-toend system TextEvaluator (Sheehan et al., 2013), and linear models (Vajjala and Meurers, 2014; Petersen and Ostendorf, 2009; Schwarm and Ostendorf, 2005). These systems leverage knowledgebased features to train regression or classification models. Most systems are trained on literary and generic texts, since analysis of text difficulty is usually tied to language teaching. Existing approaches for automated text complexity analysis pose two issues: 1) systems using knowledge based features typically work better for longer texts (Vajjala and Meurers, 2014), and 2) complex- ity estimates are less accurate for informational texts such as science (Sheehan et al., 2013). In the context of science, technology and engineering (STEM) education, both problems are significant. Teachers in these areas have less expertise in identifying appropriate reading material for students as opposed to language teachers, and shorter texts become important when dealing with assessment questions and identifying the most difficult parts of instructional texts to modify for supporting students who are ELLs.

Our work specifically looks at ways to address these two problems. First, we propose recurrent neural network (RNN) architectures for estimating linguistic complexity, using text as input without feature engineering. Second, we specifically train on science and other informational texts, using the grade level of text as a proxy for linguistic complexity and dividing grades k-12 into 6 groups. We explore four different RNN architectures in order to identify aspects of text which contribute more to complexity, with a novel structure introduced to account for cross-sentence context. Experimental results show that when specifically trained for informational texts, RNNs can accurately predict text difficulty for shorter science texts. The models also generalize to other types of texts, but perform slightly worse than feature-based regression models on a mix of genres for texts longer than 100 words. We use attention with all models, both to improve accuracy, and as a tool to visualize important elements of text contributing to linguistic complexity. The key contributions of the work include new neural network architectures for characterizing documents and experimental results demonstrating good performance for predicting reading level of short science texts.

The rest of the paper is organized as follows: section 2 looks at existing work on automated readability analysis and introduces RNN architec- 
tures we build on for this work. Section 3 lays out the data sources, section 4 covers proposed models, and section 5 presents results. Discussion and concluding remarks follow in sections 6 and 7.

\section{Background}

Studies have shown that language difficulty of instructional materials and assessment questions impacts student performance, particularly for language learners (Hickendorff, 2013; Abedi and Lord, 2001; Abedi, 2006). This has lead to extensive work on readability analysis, some of which is explored here. The second part of this section looks at work that leverages RNNs in automatic text classification tasks and the use of attention with RNNs.

\subsection{Automated Readability Analysis}

Traditional reading metrics including FleschKincaid (Kincaid et al., 1975) and Coleman-Liau index (Coleman and Liau, 1975) are often used to assess a text for difficulty. These metrics utilize surface features such as average length of sentences and words, or word lists (Chall and Dale, 1995). The development of automated text analysis systems has made it possible to leverage additional linguistic features, as well as conventional reading metrics, to estimate text complexity quantified as reading level. NLP tools can be used to extract a variety of lexical, syntactic and discourse features from text, which can then be used with traditional features as input to models for predicting reading level. Some of the models include statistical language models (Collins-Thompson and Callan, 2004), support vector machine classifiers (Schwarm and Ostendorf, 2005; Petersen and Ostendorf, 2009), and logistic regression (Feng et al., 2010). Text coherence has also been explored as a predictor of difficulty level in (Graesser et al., 2004), with an extended feature set that includes syntactic complexity and discourse in addition to coherence (Graesser et al., 2011).

A study conducted in (Nelson et al., 2012) indicates that metrics that incorporate a large set of linguistic features perform better at predicting text difficulty level; the metrics were specifically tested on the Common Core Standards (CCS) texts. ${ }^{1}$ Features from second language acquisition complexity measures were used in (Vajjala and Meurers, 2012) to improve readability assessment. This

\footnotetext{
${ }^{1}$ http://www.corestandards.org/
}

feature set was further extended to include morphological, semantic and psycholinguistic features to build a readability analyzer for shorter texts (Vajjala and Meurers, 2014). A tool specifically built for text complexity analysis for teaching and assessing is the TextEvaluator ${ }^{\mathrm{TM}}$. While knowledgebased features offer interpretability, a drawback is that if the text being analyzed is short, the feature vector is sparse, and prediction accuracy drops (Vajjala and Meurers, 2014). This is particularly true for assessment questions, which are shorter than the samples most models are trained on.

Generally, for any text classification task, the type of text used for training the model is important in terms of how well it performs; training on more representative text tends to improve performance. The work in (Sheehan et al., 2013) shows that traditional readability measures underestimate the reading level of literary texts, and overestimate that of informational texts, such as history, science and mathematics articles. This is due, in part, to the vocabulary specific to the genre. Science texts have longer words, though they may be easier to infer from context. Literary texts, on the other hand, might have simpler words, but more complicated sentence structure. The work demonstrated that more accurate grade level estimates can be obtained by two stage classification: i) classify the text as either literary, informational, or mixed, and then ii) use a genre-dependent analyzer to estimate the level. In an analysis on how well a model trained on news and informational articles generalizes to the categories in CCS, the work in (Vajjala and Meurers, 2014) shows better performance on informational genre than literary texts. Training on more representative text, however, requires genre-specific annotated data.

\subsection{Text Classification with RNNs}

Recurrent neural networks (RNNs) are adept at learning text representations, as demonstrated by language modeling (Mikolov et al., 2010) and text classification tasks (Yogatama et al., 2017). Additional RNN structures have been proposed for improved representation, including tree LSTMs (Tai et al., 2015) and a hierarchical RNN (Yang et al., 2016). In addition, hierarchical models have been proposed to better represent document structure (Yang et al., 2016).

Attention mechanisms were introduced to improve neural machine translation tasks (Bahdanau et al., 2014), and have also been shown to im- 
prove the performance of text classification (Yang et al., 2016). In machine translation, attention is computed over the source sequence when predicting the words in the target sequence. This "context" attention is based on a score computed between the target hidden state $h_{t}$ and a subset of the source hidden states $h_{s}$. The score can be computed in several ways, of which a general form is $\operatorname{score}\left(h_{t}, h_{s}\right)=h_{t}^{T} W_{\alpha} h_{s}^{T}$ (Luong et al., 2015).

Attention has also been used for a variety of other language processing tasks. In particular, for text classification, attention weights are learned that target the final classification decision. This approach is referred to as "self attention" in (Lin et al., 2017), but will be referred to here as "task attention." The hierarchical RNN in (Yang et al., 2016) uses task attention mechanisms at both word and sentence levels. Since our work builds on this model, it is described in further detail in section 4. In addition, we propose extensions of the hierarchical RNN that leverage attention in different ways, including combining the concept of context attention from machine translation with task attention to capture interdependence of adjoining sentences in a document.

\section{Data}

For our work we consider grade level as a proxy for linguistic complexity. Within a grade level, there is variability across different genres, which students are expected to learn. Since there is no publicly available data set for estimating grade level and text difficulty aimed at informational texts, we created a corpus using online science, history and social studies textbooks. The textbooks are written for either specific grades, or for a grade range, e.g. grades 6-8. There are a total of 44 science textbooks and 11 history and social studies textbooks, distributed evenly across grades K-12. Given the distribution of textbooks for each grade level, we decide to classify into one of six grade bands: $\mathrm{K}-1,2-3,4-5,6-8,9-10$ and 11-12. Because of our interest in working with short texts, we split the books into paragraphs, using end line as the delimiter. ${ }^{2}$ In addition to the textbooks, we also used the WeeBit corpus (Vajjala and Meurers, 2012) for training, again split into paragraphs.

\footnotetext{
${ }^{2}$ In splitting the text into paragraphs, we are implicitly assuming that all paragraphs have the same linguistic complexity as the textbook, which is probably not the case. Thus, there will be noise in both the training and test data, so some variation in the predicted levels is to be expected.
}

\begin{tabular}{c|c|c}
\hline \hline Grade Level & All chapters & $\begin{array}{c}\text { Test set } \\
\text { chapters }\end{array}$ \\
\hline K-1 & 25 & - \\
$2-3$ & 22 & 2 \\
$4-5$ & 53 & 9 \\
$6-8$ & 165 & 12 \\
$9-10$ & 48 & 5 \\
$11-12$ & 28 & 3 \\
\hline \hline
\end{tabular}

Table 1: Chapter-based test data split

We have three different sources of test data: i) the CCS appendix B texts, ii) a subset of the online texts that we collected, ${ }^{3}$ and iii) a collection of science assessment items.

The CCS appendix B data is of interest because it has been extensively used for evaluating linguistic complexity models, e.g. in (Sheehan et al., 2013; Vajjala and Meurers, 2014). It includes both informational and literary texts. We use document-level samples from the CCS data for comparison to prior work, and paragraph-level samples to provide a more direct comparison to the information test data we created.

For the informational texts, we selected chapters from multiple open source texts. Since we had so few texts at the K-1 level, the test data only included texts from higher grade levels, as shown in table 1. The paragraphs in these chapters were randomly assigned to test and validation sets.

To assess the models on stand alone texts, we assembled a corpora of science assessment questions from (Khot et al., 2015; Clark et al., 2018), AI2 Science Questions Mercury, ${ }^{4}$ and AI2 Science Questions v2.1 (October 2017). ${ }^{5}$ This test set includes 5470 questions for grades 6-8 from sources including standardized state and national tests. The average length of a question is 49 words.

For training, two data configurations were used. When testing on the CCS data and the science assessment questions, there is no concern about overlap between training and test data, so all text can be used for training. We held out $10 \%$ of this data for analysis, and the remaining text is used for the $D_{1}$ training configuration. Data statistics are given in table 2. About $20 \%$ of the training sam-

\footnotetext{
${ }^{3}$ Available at https://tinyurl.com/yc59hlgj.

${ }^{4}$ http: / /data.allenai.org/ ai2-science-questions-mercury/

${ }^{5}$ http://data.allenai.org/ ai2-science-questions/
} 


\begin{tabular}{c|c|c}
\hline \hline $\begin{array}{c}\text { Grade } \\
\text { Level }\end{array}$ & $\begin{array}{c}\text { Train } \\
\text { Samples }\end{array}$ & $\begin{array}{c}\text { Mean } \\
\text { Length }\end{array}$ \\
\hline K-1 & 739 & 24.42 \\
$2-3$ & 723 & 62.05 \\
$4-5$ & 4570 & 63.82 \\
$6-8$ & 15940 & 74.79 \\
$9-10$ & 3051 & 68.24 \\
$11-12$ & 2301 & 75.28 \\
\hline \hline
\end{tabular}

Table 2: Training data $\left(D_{1}\right)$ with mean length of text in words

ples (5152) are from WeeBit, spread across grades 2-12. For testing on all three sets, we defined a training configuration $D_{2}$ that did not include any text from chapters overlapping with the test data, so there training set is somewhat smaller than for $D_{1}$, except for grades $\mathrm{K}-1$. The same WeeBit training data was included in both cases.

For the elementary grade levels, we have much less data than for middle school, and for high school, we have substantial training data with coarser labels (grades 9-12). To work around both issues, we first used all training samples to train the RNN to predict one of four labels (grades K-3, 4-5, 6-8 and 9-12). We then used the training data with fine labels to train to predict one of six labels. This approach was more effective than alternating the training.

\section{Models for Estimating Linguistic Complexity}

This section introduces the four RNN structures for linguistic complexity estimation, including: a sequential RNN with task attention, a hierarchical attention network, and two proposed extensions of the hierarchical model using multi-head attention and attention over bidirectional context. In all cases, the resulting document vector is used in a final stage of ordinal regression to predict linguistic complexity. All systems are trained in an end-toend fashion.

\subsection{Sequential RNN}

The basic RNN model we consider is a sequential RNN with task attention, where the entire text in a paragraph or document is taken as a sequence. For a document $t_{i}$ with words $K$ words $w_{i k} k \in$ $\{1,2, \ldots, K\}$, a bidirectional GRU is used to learn representation for each word $h_{i k}$, using a forward run from $w_{i 1}$ to $w_{i K}$, and a backward run from $w_{i K}$ to $w_{i 1}$.

$$
\begin{gathered}
\overrightarrow{h_{i k}}=\overrightarrow{G R U}\left(w_{i k}\right) \\
\overleftarrow{h_{i k}}=\overleftarrow{G R U}\left(w_{i k}\right) \\
h_{i k}=\left[\overrightarrow{h_{i k}}, \overleftarrow{h_{i k}}\right]
\end{gathered}
$$

Attention is computed over the entire sequence $\alpha_{i k}$, and used to compute the document representation $v_{i}^{s e q}$ :

$$
\begin{gathered}
u_{i k}=\tanh \left(W_{s} h_{i k}+b_{s}\right) \\
\alpha_{i k}=\frac{\exp \left(u_{i k}^{T} u_{s}\right)}{\sum_{i k} \exp \left(u_{i k}^{T} u_{s}\right)} \\
v_{i}^{s e q}=\sum_{k} \alpha_{i k} h_{i k}
\end{gathered}
$$

The document vector is used to predict reading level. Since the grade levels are ordered categorical labels, we implement ordinal regression using the proportional odds model (McCullagh, 1980). For the reading level labels $j \in\{1,2, \ldots, J\}$, the cumulative probability is modeled as

$$
P\left(y \leq j \mid v_{i}^{\text {seq }}\right)=\sigma\left(\beta_{j}-w_{\text {ord }}^{T} v_{i}^{\text {seq }}\right),
$$

where $\sigma($.$) is the sigmoid function, and \beta_{j}$ and $w_{\text {ord }}$ are estimated during training by minimizing the negative log-likelihood

$$
\begin{gathered}
\mathcal{L}_{\text {ord }}=-\sum_{i} \log \left(\sigma\left(\beta_{j(i)}-w_{\text {ord }}^{T} v_{i}^{\text {seq }}\right)-\right. \\
\left.\sigma\left(\beta_{j(i)-1}-w_{\text {ord }}^{T} v_{i}^{\text {seq }}\right)\right) .
\end{gathered}
$$

\subsection{Hierarchical RNN}

While a sequential RNN has the capacity to capture discourse across sentences, it does not capture document structure. Therefore, we also explored the hierarchical attention network for text classification from (Yang et al., 2016). The model builds a vector representation $v_{i}$ for each document $t_{i}$ with $L$ sentences $s_{l}, l \in\{1,2, . ., L\}$, each with $T_{l}$ words $w_{l t}, t \in\left\{1,2, \ldots, T_{l}\right\}$. The first level of the hierarchy takes words as input and learns a representation for each word $h_{l t}$ using a bidirectional GRU. Task attention at the word level $\alpha_{l t}$ highlights words important for the classification task, and is computed using the word level context vector $u_{w}$. The word representations are then averaged using attention weights to form a sentence representation $s_{l}$

$$
\begin{gathered}
\alpha_{l t}=\frac{\exp \left(u_{l t}^{T} u_{w}\right)}{\sum_{t} \exp \left(u_{l t}^{T} u_{w}\right)} \\
s_{l}=\sum_{t} \alpha_{l t} h_{l t},
\end{gathered}
$$


where $u_{l t}=\tanh \left(W_{w} h_{l t}+b_{w}\right)$ is a projection of the target hidden state for learning word-level attention. The second level of the hierarchy takes the sentence vectors as input, learns representation $h_{l}$ for them using a bidirectional GRU. Using a method similar to the word-level attention, a document representation $v_{i}$ is created using sentencelevel task attention $\alpha_{l}$ which is computed using the sentence level context vector $u_{s}$

$$
\begin{gathered}
\alpha_{l}=\frac{\exp \left(u_{l}^{T} u_{s}\right)}{\sum_{l} \exp \left(u_{l}^{T} u_{s}\right)} \\
v_{i}=\sum_{l} \alpha_{l} h_{l},
\end{gathered}
$$

where $u_{l}=\tanh \left(W_{s} h_{l}+b_{s}\right)$ is analogous to $u_{l t}$ at the sentence level. The word- and sentence-level context vectors, $u_{w}$ and $u_{s}$, as well as $W_{w}, W_{s}, b_{w}$ and $b_{s}$, are learned during training.

\subsection{Multi-Head Attention}

Work has shown that having multiple attention heads improves neural machine translation tasks (Vaswani et al., 2017). To capture multiple aspects contributing to text complexity, we learn two sets of word level task attention over the word level GRU output. These two sets of sentence vectors feed into separate sentence-level GRUs to give us two document vectors by averaging using task attention weights at the sentence level. The document vectors are then concatenated to form the document representation. The multi-head attention RNN is shown in figure 1 .

\subsection{Hierarchical RNN with Bidirectional Context}

The hierarchical model is designed for representing document structure, however, the sentences within a document are encoded independently. To capture information across sentences, we extend the concept of context attention used in machine translation, using it to learn context vectors for adjoining sentences. We extend the hierarchical RNN by introducing bi-directional context with attention. Using the word level GRU output, a "look-back" context vector $c_{l-1}\left(w_{l t}\right)$ is calculated using context attention over the preceding sentence, and a "look-ahead" context vector $c_{l+1}\left(w_{l t}\right)$ using context attention over the following sentence for each word in the current sentence.

$$
\begin{gathered}
\alpha_{(l-1) t}\left(w_{l t}\right)=\frac{\exp \left(\operatorname{score}\left(h_{l t}, h_{(l-1) t}\right)\right)}{\sum_{t^{\prime}} \exp \left(\operatorname{score}\left(h_{l t}, h_{(l-1) t^{\prime}}\right)\right)} \\
c_{l-1}\left(w_{l t}\right)=\sum_{t^{\prime}} \alpha_{(l-1) t^{\prime}}\left(w_{l t}\right) h_{(l-1) t^{\prime}}
\end{gathered}
$$

$$
\begin{gathered}
\alpha_{(l+1) t}\left(w_{l t}\right)=\frac{\exp \left(\operatorname{score}\left(h_{l t}, h_{(l+1) t}\right)\right)}{\sum_{t^{\prime}} \exp \left(\operatorname { s c o r e } \left(h_{l t}, h_{\left.\left.(l+1) t^{\prime}\right)\right)}\right.\right.} \\
c_{l+1}\left(w_{l t}\right)=\sum_{t^{\prime}} \alpha_{(l+1) t^{\prime}}\left(w_{l t}\right) h_{(l+1) t^{\prime}}
\end{gathered}
$$

where $\operatorname{score}\left(h_{l t}, h_{k t}\right)=h_{l t} W_{\alpha} h_{k t}^{T}$ and a single $W_{\alpha}$ is used for computing the score in both directions. The context vectors are concatenated with the hidden state to form the new hidden state $h_{l t}^{\prime}$.

$$
h_{l t}^{\prime}=\left[c_{l-1}\left(w_{l t}\right), h_{l t}, c_{l+1}\left(w_{l t}\right)\right]
$$

The rest of the structure is the same as a hierarchical RNN, using equations 9-12 with $h_{l t}^{\prime}$ instead of $h_{l t}$. Figure 2 shows the structure for calculating 'look-back" context.

\subsection{Implementation Details}

The implementation is done via the Tensorflow library (Abadi et al., 2016). ${ }^{6}$ All RNNs use GRUs (Cho et al., 2014) with layer normalization (Ba et al., 2016), trained using Adam optimizer (Kingma and $\mathrm{Ba}, 2014$ ) with a learning rate of 0.001. Regularization was done via drop out. The validation set was used to do hyper-parameter tuning, with a grid search over drop out rate, number of epochs, and hidden dimension of GRU cells. Good result for all four architectures are obtained with a batch size of 10 , a dropout rate of 0.5-0.7, a cell size of 75-250 for the word-level GRU, and a cell size of 40-75 for the sentence-level GRU. For the RNN, we also trained a version with a larger word-level hidden layer cell size of 600 .

Pre-trained Glove embeddings ${ }^{7}$ are used for all models (Pennington et al., 2014), using a vocabulary size of $65000-75000 .{ }^{8}$ The out of vocabulary (OOV) percentage on the CCS test set was 3\%, and on the informational test set was $0.5 \%$. All OOV words were mapped to an 'UNK' token. The text was lower-cased, and split into sentences for the hierarchical models using the natural language toolkit (NLTK) (Loper and Bird, 2002).

\section{Results and Analysis}

We test our models on the two science test sets, as well as on the CCS appendix B document level texts and a paragraph-level version of these texts. We also evaluated the best performing

\footnotetext{
${ }^{6}$ The code and trained models are available at https: //github.com/Farahn/Liguistic-Complexity.

${ }^{7}$ http://nlp.stanford.edu/data/glove. 840 B.300d.zip

${ }^{8}$ In vocabulary words not present in Glove had randomly initialized word embeddings.
} 


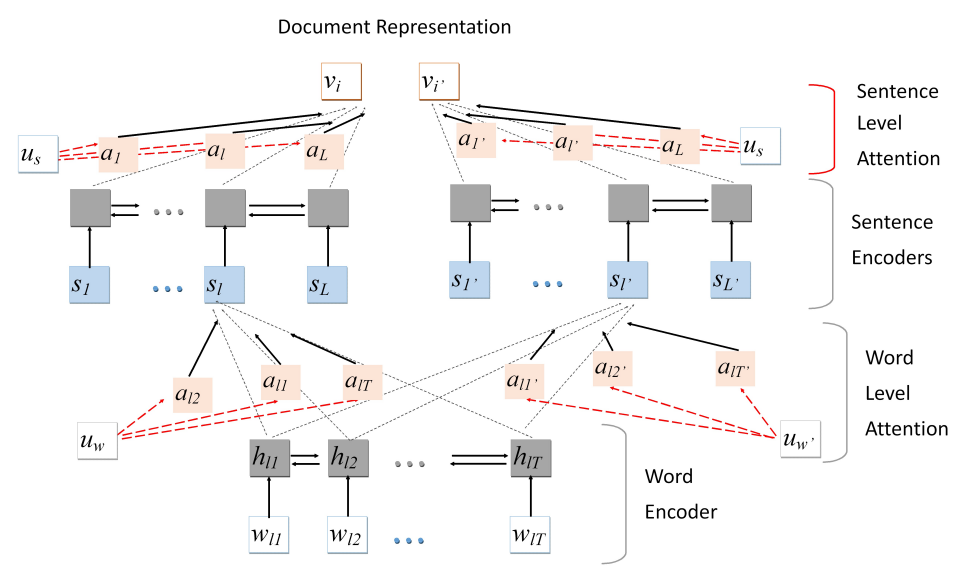

Figure 1: RNN with Multi-Head Attention

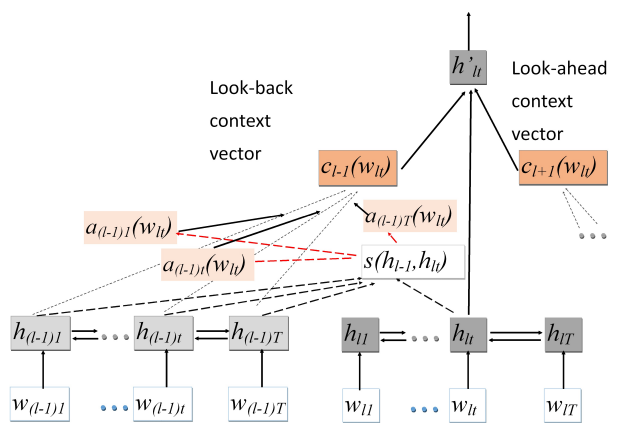

Figure 2: RNN with Bidirectional Context and Attention

model on the middle school science questions data set. Since both the true reading level and predicted levels are ordered variables, we use Spearman's rank correlation as the evaluation metric to capture the monotonic relation between the predictions and the true levels.

As a baseline, we use the WeeBit linear regression system (Vajjala and Meurers, 2014). The WeeBit system uses knowledge-based features as input to a linear regression model to predict reading level as a number between 1 and 5.5, which maps to text appropriate for readers 7-16 years of age. The feature set includes parts-of-speech (e.g. density of different parts-of-speech), lexical (e.g. measurement of lexical variation), syntactic (e.g. the number of verb phrases), morphological (e.g. ratio of transitive verbs to total words) and psycholinguistic (e.g. age of acquisition) features. There are no features related to discourse, thus it is possible to compute features for sentence level texts. The system was trained on a subset of the data that our system was trained on, so it is at a disadvantage. We did not have the capability to retrain the system.

\subsection{Results by Genre}

Results for the different models:

- sequential RNN with self attention (RNN),

- large sequential RNN with self attention (RNN 600),

- hierarchical RNN with attention at the word and sentence level (HAN),

- hierarchical RNN with bidirectional context and attention (BCA), and

- multi-head attention (MHA)

are shown in table 3, together with the results for the WeeBit system which has state-of-the-art results on the CCS documents. For the CCS data, both $D_{1}$ and $D_{2}$ training configurations are used for the neural models; only $D_{2}$ is used for the informational test set. For all of these models the hidden layer dimension for the word level was between 125 and 250. We also trained a sequential RNN with a larger hidden layer dimension of 600 .

The HAN does better for document level samples than a sequential RNN; the converse is true for paragraph level texts. The RNN with a larger hidden layer dimension performs better for longer texts, while the performance for smaller dimension RNN deteriorates with increasing text length. The BCA model seems to generalize to longer documents and new genres better than the other neural networks.

Figure 3 shows the error distribution for $\mathrm{BCA}\left(D_{1}\right)$ in terms of distance from true prediction broken down by genre on the 168 CCS documents. The category of informational texts is often over 


\begin{tabular}{l|c|ccccccc}
\hline \hline Test Set & Model & Samples & WeeBit & RNN & RNN 600 & HAN & BCA & MHA \\
\hline CCS Document & $D_{1}$ & 168 & 0.69 & 0.28 & 0.43 & 0.47 & 0.55 & 0.42 \\
\hline CCS Paragraphs & $D_{1}$ & 1532 & 0.36 & 0.30 & 0.25 & 0.29 & 0.32 & 0.28 \\
\hline CCS Document & $D_{2}$ & 168 & 0.69 & 0.34 & 0.38 & 0.43 & 0.48 & 0.43 \\
\hline CCS Paragraphs & $D_{2}$ & 1532 & 0.36 & 0.27 & 0.26 & 0.24 & 0.30 & 0.29 \\
\hline $\begin{array}{l}\text { Informational } \\
\text { Paragraphs }\end{array}$ & $D_{2}$ & 1361 & 0.22 & 0.51 & 0.60 & 0.60 & 0.62 & 0.60 \\
\hline \hline
\end{tabular}

Table 3: Results (Spearman Rank Correlation)

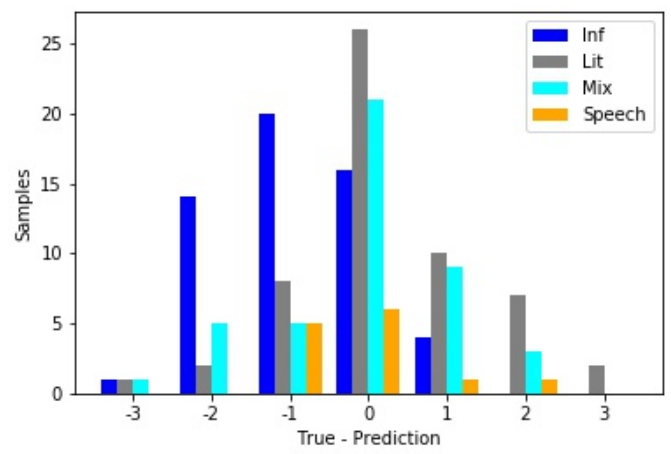

Figure 3: Error distribution for the CCS documents $\operatorname{BCA}\left(D_{1}\right)$

predicted, which we hypothesize is roughly due to specific articles related to the United States history and constitution. The only training data for our models with that subject is in the grades 6-8 and 9-12 categories. The performance for literary and mixed texts, on the other hand, is roughly unbiased; this shows that the model is better at generalizing to non-informational texts, even when there are no literary text samples in the training data.

\subsection{Results by Length}

Figures 4 and 5 show the performance of our models and the WeeBit model as a function of document length, both on the informational paragraphs test set and the CCS paragraph level test set. The results indicate that for shorter texts, particularly under 100 words, neural models tend to do better. Even for a mixture of genres, the model with bidirectional context performs better than the featurebased regression model, as shown in figure 5 .

It is likely that the WeeBit results results on shorter texts would improve if trained on the same training set that is used for the neural models. However, we hypothesize that the feature-based approach is less well suited for shorter documents because the feature vector will be more sparse.

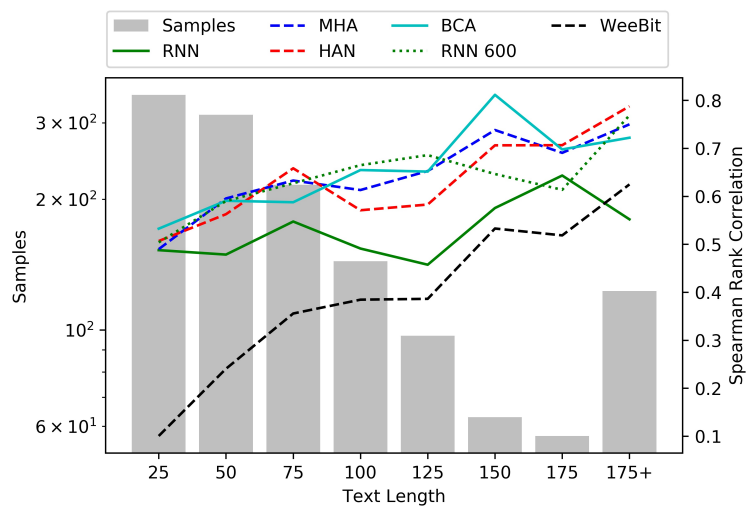

Figure 4: Performance vs. text length for informational paragraphs $\operatorname{BCA}\left(D_{2}\right)$

Comparing the CCS document- and paragraphlevel test sets, the average percentage of features that are zero-valued is $28 \%$ for document-level texts and $44 \%$ for paragraph-level texts. The most sparse vectors are $40 \%$ and $81 \%$ for document and paragraph-level texts, respectively.

\subsection{Results for Science Assessment Questions}

Finally, we apply both the baseline WeeBit system and our best model (BCA trained on $D_{1}$ ) to the set of 5470 grade 6-8 science questions. The results are shown in figures 6 and 7, where the grade 68 category (ages 11-14) corresponds to predicted level 3 for BCA and predicted level 4 for WeeBit. The results indicate that BCA predictions are better aligned with human rankings than the baseline. As expected, grade 6 questions more likely to be predicted as less difficult than grade 8 questions.

\subsection{Attention Visualization}

Attention can help provide insight into what the model is learning. In the analyses here, all attention values are normalized by dividing by the highest attention value in the sentence/document to account for different sequence lengths.

Figure 8 shows the word-level attention for the 


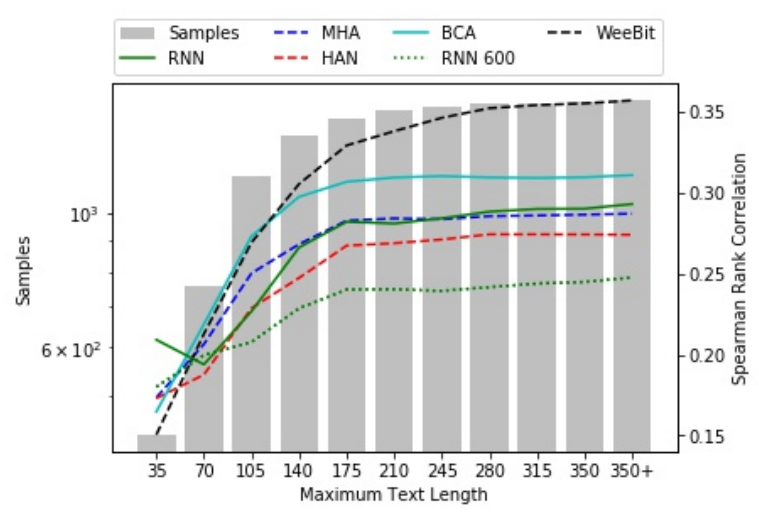

Figure 5: Performance vs. maximum text length for CCS paragraphs $\operatorname{BCA}\left(D_{1}\right)$

BCA and HAN for a sample text from the science assessment questions test set. (Attention weights in the figure are smoothed to reflect the fact that a word vector from a biLSTM reflects the word's context.) The results show that attention weights are more sparse for HAN than for BCA. At the sentence level (not shown here), the BCA sentence weights tend to be more uniformly distributed, whereas HAN weights are again more selective.

Another aspect of the attention is that a word does not have the same attention level for all occurrences in a document. We look at maximum and minimum values of attention as a function of word frequency for each grade band, shown in figure 9 for grade 6-8 science assessment questions.

The pattern is similar for each grade band in the validation and test sets. The minimum attention values assigned to a word drop with increasing word frequency, while the maximum values increase. This suggests that the attention weights are more confident for more frequent words, such as of. Words like fusion and $\mathrm{m} / \mathrm{s}$ get high max-

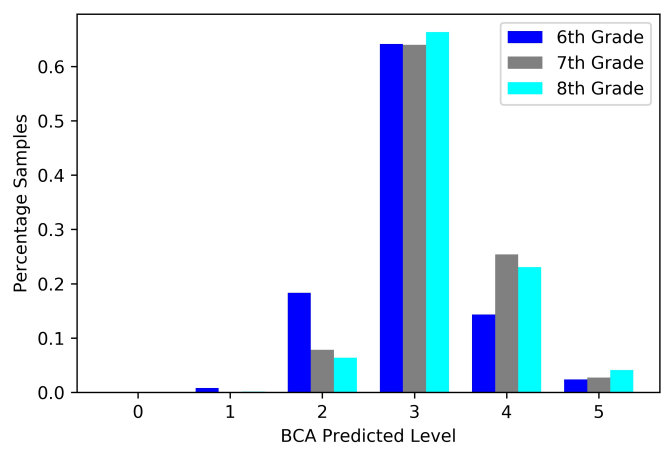

Figure 6: BCA predicted levels for middle school science assessment questions imum attention values, despite not being as high frequency as words like of and the. This may indicate that they are likely to contribute to linguistic complexity. The fact that transformation has a high minimum is also likely an indicator of its importance. For HAN without bidirectional context, a similar visualization shows that while the trend is similar, the attention weights typically tend to be lower, both for minimum and maximum values.

We find that sentence-end tokens (period, exclamation and question mark) have high average attention weight, ranging from 0.54 to 0.81 , while sentence-internal punctuation (comma, colon and semicolon) get slightly lower weights, ranging from 0.20 to 0.47 . The trend is similar for all grades. These high attention values might be due to punctuation serving as a proxy for sentence structure. It is interesting to note that the question mark gets higher minimum attention value than period, despite being high frequency. It may be that questions carry information that is particularly relevant to informational text difficulty.

\section{Discussion}

Our work differs from existing models that estimate text difficulty since we do not use engineered features. There are advantages and disadvantages to both approaches, which we briefly discuss here. Models using engineered features based on research on language acquisition offer interpretability and insight into which specific linguistic features are contributing to text difficulty. An additional advantage of using engineered features in a regression or classification model is that less training data is required.

However, given both the evolving theories in

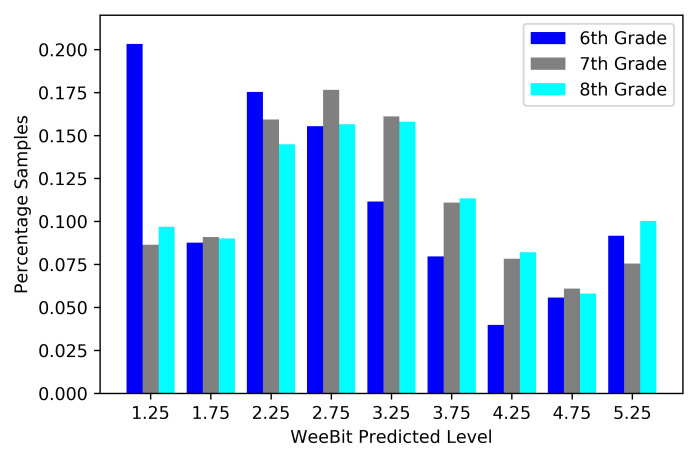

Figure 7: WeeBit predicted levels for middle school science assessment questions 
a physicist wants to determine the speed a car must reach to jump over a ramp . the physicist conducts three trials . in trials two and three, the speed of the car is increased by 20 miles per hour . what is the physicist investigating when he changes the speed ? ( a ) the control ( b ) the hypothesis statement (c ) the dependent ( responding ) variable ( $d$ ) the independent ( manipulated ) variable

a physicist wants to determine the speed a car must reach to jump over a ramp . the physicist conducts three trials . in trials two and three, the speed of the car is increased by 20 miles per hour. what is the physicist investigating when he changes the speed ? ( a ) the control ( b ) the hypothesis statement (c ) the dependent ( responding ) variable ( $\mathrm{d}$ ) the independent ( manipulated ) variable

Figure 8: Word level attention visualization for BCA (top) and HAN (bottom) for a middle school science assessment question

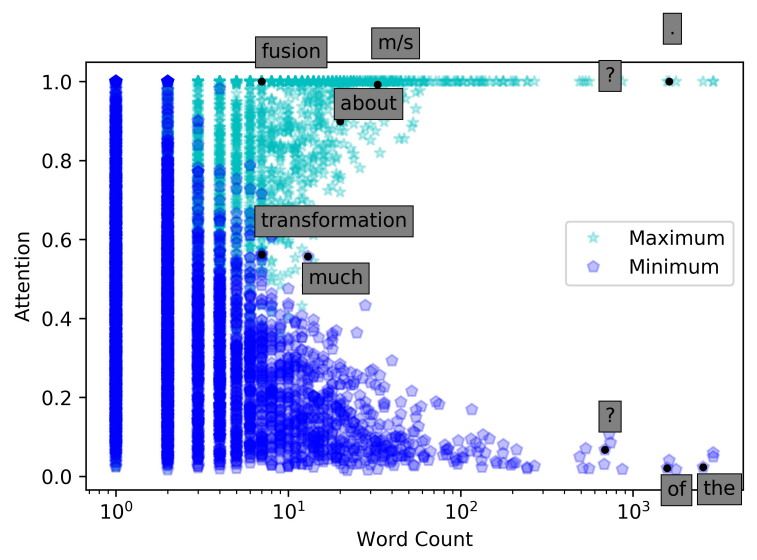

Figure 9: Maximum and minimum values of attention as a function of word count for BCA

language acquisition and the large number of variables that impact second language acquisition, the methodologies used in language acquisition research have certain limitations. For example, the number of variables that can be considered in a study is practically limited, the sample population is often small, and the question of qualitative vs. quantitative methodologies used can influence outcomes (more details in (Larsen-Freeman and Long, 2014; Mitchell et al., 2013)). These limitations can carry into the feature engineering process. Using a model with text as input ensures that these constraints are not inherently part of the model; the performance of the system is not limited by the features provided. Of course, performance is limited by the training data, both in terms of the cost of collection and any biases inherent in the data. In addition, with advances in neural architectures such as attention modeling, there may be opportunities for identifying specific aspects of texts that are particularly difficult, though research in this direction is still in early stages.

\section{Conclusion}

In summary, this work explored different neural architectures for linguistic complexity analysis, to mitigate issues with accuracy of systems based on engineered features. Experimental results show that it is possible to achieve high accuracy on texts shorter than 100 words using RNNs with attention. Using hierarchical structure improves results, particularly with attention models that leverage bidirectional sentence context. Testing on a mix of genres shows that the best neural model can generalize to subjects beyond what it is trained on, though it performs slightly worse than a featurebased regression model on texts longer than 100 words. More training data from other genres will likely reduce the performance gap. Analysis of attention weights can provide insights into which phrases/sentences are important, both at the aggregate and sample level. Developing new methods for analysis of attention may be useful both for improving model performance and for providing more interpretable results for educators.

Two aspects not considered in this work are explicit representation of syntax and discourse structure. Syntax can be incorporated by concatentating word and dependency embeddings at the token level. Our BCA model was designed to capture cross-sentence coherence and coordination, but it may be useful to extend the hierarchy for longer documents and/or introduce explicit models of the types of discourse features used in Coh-Metrix (Graesser et al., 2004).

\section{Acknowledgments}

We thank Dr. Meurers, Professor University of Tubingen, and Dr. Vajjala-Balakrishna, Assistant Professor Iowa State University, for sharing the WeeBit training corpus, their trained readability assessment model and the Common Core test corpus.

\section{References}

Martín Abadi, Ashish Agarwal, Paul Barham, Eugene Brevdo, Zhifeng Chen, Craig Citro, Greg S Corrado, Andy Davis, Jeffrey Dean, Matthieu Devin, et al. 2016. Tensorflow: Large-scale machine learning on 
heterogeneous distributed systems. arXiv preprint arXiv:1603.04467.

Jamal Abedi. 2006. Psychometric issues in the ELL assessment and special education eligibility. Teachers College Record, 108(11):2282.

Jamal Abedi and Carol Lord. 2001. The language factor in mathematics tests. Applied Measurement in Education, 14(3):219-234.

Jimmy Lei Ba, Jamie Ryan Kiros, and Geoffrey E Hinton. 2016. Layer normalization. arXiv preprint arXiv:1607.06450.

Dzmitry Bahdanau, Kyunghyun Cho, and Yoshua Bengio. 2014. Neural machine translation by jointly learning to align and translate. arXiv preprint arXiv:1409.0473.

Jeanne Sternlicht Chall and Edgar Dale. 1995. Readability revisited: The new Dale-Chall readability formula. Brookline Books.

Kyunghyun Cho, Bart Van Merriënboer, Caglar Gulcehre, Dzmitry Bahdanau, Fethi Bougares, Holger Schwenk, and Yoshua Bengio. 2014. Learning phrase representations using rnn encoder-decoder for statistical machine translation. arXiv preprint arXiv:1406.1078.

Peter Clark, Isaac Cowhey, Oren Etzioni, Tushar Khot, Ashish Sabharwal, Carissa Schoenick, and Oyvind Tafjord. 2018. Think you have solved question answering? try arc, the ai 2 reasoning challenge. arXiv preprint arXiv:1803.05457.

Meri Coleman and Ta Lin Liau. 1975. A computer readability formula designed for machine scoring. Journal of Applied Psychology, 60(2):283.

Kevyn Collins-Thompson and James P. Callan. 2004. A language modeling approach to predicting reading difficulty. In Human Language Technology Conference of the North American Chapter of the Association for Computational Linguistics, HLT-NAACL 2004, Boston, Massachusetts, USA, May 2-7, 2004, pages 193-200.

Lijun Feng, Martin Jansche, Matt Huenerfauth, and Noémie Elhadad. 2010. A comparison of features for automatic readability assessment. In Proceedings of the 23rd International Conference on Computational Linguistics: Posters, pages 276-284. Association for Computational Linguistics.

Arthur C. Graesser, Danielle S. McNamara, and Jonna M. Kulikowich. 2011. Coh-metrix. Educational Researcher, 40(5):223-234.

Arthur C Graesser, Danielle S McNamara, Max M Louwerse, and Zhiqiang Cai. 2004. Coh-metrix: Analysis of text on cohesion and language. Behavior Research Methods, 36(2):193-202.
Marian Hickendorff. 2013. The language factor in elementary mathematics assessments: Computational skills and applied problem solving in a multidimensional irt framework. Applied Measurement in Education, 26(4):253-278.

Tushar Khot, Niranjan Balasubramanian, Eric Gribkoff, Ashish Sabharwal, Peter Clark, and Oren Etzioni. 2015. Markov logic networks for natural language question answering. arXiv preprint arXiv:1507.03045.

J Peter Kincaid, Robert P Fishburne Jr, Richard L Rogers, and Brad S Chissom. 1975. Derivation of new readability formulas (automated readability index, fog count and flesch reading ease formula) for navy enlisted personnel. Technical report, Naval Technical Training Command Millington TN Research Branch.

Diederik P Kingma and Jimmy Ba. 2014. Adam: A method for stochastic optimization. arXiv preprint arXiv:1412.6980.

Diane Larsen-Freeman and Michael H Long. 2014. An introduction to second language acquisition research. Routledge.

Zhouhan Lin, Minwei Feng, Cicero Nogueira do Santos, Mo Yu, Bing Ziang, Bowen Zhou, and Yoshua Bengio. 2017. A structured self-attentive sentence embedding. In Proc. ICLR.

Edward Loper and Steven Bird. 2002. Nltk: The natural language toolkit. In Proceedings of the ACL-O2 Workshop on Effective tools and methodologies for teaching natural language processing and computational linguistics-Volume 1, pages 63-70. Association for Computational Linguistics.

Minh-Thang Luong, Hieu Pham, and Christopher D Manning. 2015. Effective approaches to attentionbased neural machine translation. arXiv preprint arXiv:1508.04025.

Peter McCullagh. 1980. Regression models for ordinal data. Journal of the royal statistical society. Series B (Methodological), pages 109-142.

Tomáš Mikolov, Martin Karafiát, Lukáš Burget, Jan Černockỳ, and Sanjeev Khudanpur. 2010. Recurrent neural network based language model. In Eleventh Annual Conference of the International Speech Communication Association.

Rosamond Mitchell, Florence Myles, and Emma Marsden. 2013. Second language learning theories. Routledge.

Jessica Nelson, Charles Perfetti, David Liben, and Meredith Liben. 2012. Measures of text difficulty: Testing their predictive value for grade levels and student performance. Council of Chief State School Officers, Washington, DC. 
Jeffrey Pennington, Richard Socher, and Christopher Manning. 2014. Glove: Global vectors for word representation. In Proceedings of the 2014 conference on empirical methods in natural language processing (EMNLP), pages 1532-1543.

Sarah E Petersen and Mari Ostendorf. 2009. A machine learning approach to reading level assessment. Computer speech \& language, 23(1):89-106.

Sarah E Schwarm and Mari Ostendorf. 2005. Reading level assessment using support vector machines and statistical language models. In Proceedings of the 43rd Annual Meeting on Association for Computational Linguistics, pages 523-530. Association for Computational Linguistics.

Kathleen M Sheehan, Michael Flor, and Diane Napolitano. 2013. A two-stage approach for generating unbiased estimates of text complexity. In Proceedings of the Workshop on Natural Language Processing for Improving Textual Accessibility, pages 49-58.

AJ Stenner, Ivan Horabin, Dean R Smith, and Malbert Smith. 1988. The lexile framework. Durham, NC: MetaMetrics.

Kai Sheng Tai, Richard Socher, and Christopher D Manning. 2015. Improved semantic representations from tree-structured long short-term memory networks. arXiv preprint arXiv:1503.00075.

Sowmya Vajjala and Detmar Meurers. 2012. On improving the accuracy of readability classification using insights from second language acquisition. In Proceedings of the Seventh Workshop on Building Educational Applications Using NLP, pages 163173. Association for Computational Linguistics.

Sowmya Vajjala and Detmar Meurers. 2014. Readability assessment for text simplification: From analysing documents to identifying sentential simplifications. ITL-International Journal of Applied Linguistics, 165(2):194-222.

Ashish Vaswani, Noam Shazeer, Niki Parmar, Jakob Uszkoreit, Llion Jones, Aidan N Gomez, Łukasz Kaiser, and Illia Polosukhin. 2017. Attention is all you need. In Advances in Neural Information Processing Systems, pages 6000-6010.

Zichao Yang, Diyi Yang, Chris Dyer, Xiaodong He, Alexander J Smola, and Eduard H Hovy. 2016. Hierarchical attention networks for document classification. In HLT-NAACL, pages 1480-1489.

Dani Yogatama, Chris Dyer, Wang Ling, and Phil Blunsom. 2017. Generative and discriminative text classification with recurrent neural networks. arXiv preprint arXiv:1703.01898. 